\title{
Insulina pancreática de ratos diabéticos tipo 1 submetidos a um protocolo de treinamento físico individualizado
}

\author{
Pancreatic insulin of type I diabetic rats subjected to an individualized \\ exercise-training protocol
}

\author{
L. Pereira de Moura, R.J. Gomes, J.A. Leme, F.A. Voltarelli, C. Ribeiro, R. Ferreira de \\ Moura, M. Barbosa de Araújo, E. Luciano, M. Rostom de Mello
}

ARTIGO ORIGINAL | ORIGINAL ARTICLE

\begin{abstract}
Dado que o diabetes mellitus é considerado um problema de saúde pública mundial, o presente estudo objetivou avaliar a tolerância à glicose oral (TTGo) e concentrações de insulina pancreática (CIP) de ratos diabéticos tipo 1 submetidos a um protocolo de treinamento físico individualizado. Foram utilizados 40 ratos Wistar adultos, onde metade foi induzida ao diabetes por meio de injeção endovenosa de aloxana $(32 \mathrm{mg} / \mathrm{kg}$ ), divididos em quatro grupos (10 por grupo): Controle Sedentário (CS), Controle Treinado (CT), Diabético Sedentário (DS) e Diabético Treinado (DT). O treinamento físico consistiu de natação, $1 \mathrm{~h} / \mathrm{dia}, 5$ dias/semana durante 8 semanas, com sobrecarga equivalente a 90\% da transição metabólica aeróbia/anaeróbia determinada no início do experimento. Os ratos dos grupos DS e DT apresentaram redução de peso corporal em relação aos controles, a qual foi menos acentuada no grupo DT. As ingestões hídrica e alimentar aumentaram nos grupos diabéticos em relação aos controles. Durante o TTGo, os valores de área sob a curva glicêmica dos grupos diabéticos foram superiores aos dos controles. O treinamento físico atenuou esta elevação. Os grupos diabéticos apresentaram CIP reduzida quando comparados aos grupos controles. O protocolo de treinamento físico empregado, embora não tenha alterado a CIP, melhorou a homeostase glicêmica e atenuou a perda de peso corporal dos animais diabéticos.

Palavras-chave: diabetes mellitus, treinamento físico, insulina pancreática
\end{abstract}

ABSTRACT

Since diabetes mellitus is considered a world public health problem, the present study aimed to evaluate oral glucose tolerance (TTGo) and pancreatic insulin concentration (PIC) of type I diabetic rats subjected to an individualized exercise training protocol. A total of 40 adult Wistar rats were used, half of which induced to diabetes by alloxan $(32 \mathrm{mg} / \mathrm{kg})$ endovenous injection, and divided into four groups (10 per group): Sedentary Control (SC), Trained Control (TC), Sedentary Diabetic (SD) and Trained Diabetic (TD). The physical training consisted of swimming, $1 \mathrm{~h} /$ day, 5 days/week during 8 weeks, supporting overload equivalent to $90 \%$ of the individual anaerobic/aerobic metabolic transition determined at the beginning of the experiment. The rats of SD and TD groups presented body weight reduction in relation to controls, which was less accentuated in TD group. Water and food ingestion increased in the diabetic groups in relation to controls. The areas under the serum glucose curve during the GTTo of diabetic groups were higher than the controls. Physical training attenuated this elevation. The diabetic groups showed reduced PIC when compared to both control groups. The physical training protocol employed improved glucose homeostasis and attenuated the body weight loss of diabetic animals but did not alter pancreatic insulin concentration.

Keywords: diabetes mellitus, physical training, pancreatic insulin

Submetido: 28.11.2009 | Aceite: 15.01.2011

Leandro Pereira de Moura, José Almeida Leme, Carla Ribeiro, Rodrigo Ferreira de Moura, Michel Barbosa de Araújo, Eliete Luciano e Maria Rostom de Mello. Departamento de Educação Física, Universidade Estadual Paulista - Rio Claro/SP, Brasil.

Ricardo José Gomes. Departamento de Educação Física, Universidade Estadual Paulista - Rio Claro/SP; Departamento de Educação Física, Universidade Federal de São Paulo, Santos/SP, Brasil.

Fabrício Azevedo Voltarelli. Departamento de Educação Física, Universidade Estadual Paulista - Rio Claro/SP; Departamento de Educação Física, Universidade Federal de Mato Grosso, Cuiabá/MT, Brasil.

Endereço para correspondência: Leandro Pereira de Moura, Departamento de Educação Física, Universidade Estadual Paulista, Av. 24ª n. ${ }^{\circ}$ 1515, Caixa Postal 199, CEP: 13506-900 Bela Vista, Rio Claro/SP, Brasil.

E-mail: leandropereiram@hotmail.com 
De acordo com a Organização Mundial de Saúde, em 2009 havia cerca de 170 milhões de diabéticos em todo o mundo e a estimativa para 2030 é que esse número dobre (Chan, Malik, \& Jia, 2009). O diabetes mellitus (DM) é caracterizado pela elevação da concentração de glicose circulante, em função da deficiência na secreção (diabetes mellitus tipo 1 - DM1) ou comprometimento na ação periférica da insulina (diabetes mellitus tipo 2 - DM2) (The Expert Committee on the Diagnosis and Classification of Diabetes Mellitus, 1997).

A insulina é um hormônio anabólico que, após interação com seu receptor de membrana específico, estimula a captação de glicose pelas células por meio de proteínas integrais de membrana, denominadas GLUTs. Tal evento regula a homeostase glicêmica, estimula a lipogênese hepática e nos adipócitos, reduz a lipólise, bem como, regula o turnover proteico (Sabetsky \& Ekblom, 2010). A caracterização do DM1, ou insulinopenia, deu-se no início da década de 1950, quando Wrenshall observou que o pâncreas de jovens com menos de 20 anos de idade, acometidos pelo diabetes, apresentava cerca de 40-50\% menos insulina em relação aos indivíduos normais (White, 1997; Wrenshall, Bogoch, \& Ritchie, 1952).

Pelo fato de existirem limitações nas pesquisas com seres humanos, modelos animais tornaram-se importantes no estudo do diabetes, por permitirem avaliações mais profundas e detalhadas das causas e das consequências da doença. Para a indução química do DM1 em ratos, a aloxana é comummente utilizada no meio científico, uma vez que essa droga age de maneira eficaz na destruição das células beta pancreáticas, causando significativa diminuição de insulina circulante nos animais (Boquist, 1977; Lenzen, 2007; Szkudelski, 2001).

A atividade física é considerada de grande importância para o tratamento do DM (American Diabetes Association, 2006). Estudos usando técnicas de biologia molecular mostram que o treinamento físico induz aumento da atividade da tirosina-quinase dos receptores de insulina, aumento da translocação dos transportadores de glicose do músculo esquelético (GLUT-4), aumento da fosforilação dos substratos do receptor de insulina (IRS-1 e IRS-2) e de sua associação à PI3-kinase independente da insulina, demonstrando que a contração muscular não necessita desse hormônio para absorver glicose circulante e promover melhora da homeostase glicêmica (Cortright \& Dohm, 1997; Luciano et al., 2002).

A identificação da intensidade de esforço no exercício é de extrema importância para a prescrição de atividade física. Um dos principais biomarcadores empregados para a determinação dessa intensidade é a concentração sanguínea de lactato. No início do exercício existe elevação da concentração de lactato, até que o metabolismo aeróbio tenha condições de suprir as necessidades energéticas do exercício. Após esse ponto, em exercício moderado, a concentração sanguínea de lactato se estabiliza, sugerindo que as taxas de produção muscular do substrato e de remoção do sangue circulante encontram-se em equilíbrio. Esta fase de equilíbrio entre produção e remoção de lactato foi denominada máxima fase estável de lactato (MFEL), e indica a maior intensidade de esforço onde o metabolismo aeróbio é o principal responsável pela energia necessária ao esforço (Harnish, Swensen, \& Pate, 2001; Mader \& Heck, 1986). A MFEL é considerada, por muitos autores, a identificação individual mais fidedigna da transição da predominância dos metabolismos aeróbio e anaeróbio durante o exercício (Beneke, 2003; Billat, Siverent, Py, Korallsztein, \& Mercier, 2003).

Estudos dos efeitos metabólicos do exercício em ratos são frequentemente questionados, devido à falta da informação sobre a intensidade do esforço executado pelos animais durante o exercício. Por esta razão, em nosso laboratório, foi estabelecido um protocolo para a determinação individual da intensidade de esforço equivalente à MFEL durante exercício de natação em ratos eutróficos (Gobatto et al., 2001).

São escassas, na literatura, informações sobre qual o melhor protocolo de exercício a 
ser empregado para portadores de DM1. Portanto, o presente estudo teve como objetivo avaliar os efeitos de um protocolo de treinamento individualizado de natação, em intensidade equivalente a $90 \%$ da MFEL, sobre tolerância à glicose e concentrações de insulina pancreática e sérica de ratos diabéticos aloxânicos.

\section{MÉTODO}

\section{Animais}

Todos os experimentos envolvendo animais, no presente estudo, seguiram as resoluções impostas pelos procedimentos para o uso científico de animais (Lei Federal n. ${ }^{\circ} 11794$, de 8 de outubro de 2008).

Foram utilizados 40 ratos adultos da linhagem Wistar, com 60 dias no início do experimento, provenientes do Biotério Central da UNESP - Universidade Estadual Paulista, campus Botucatu e mantidos no Biotério do Laboratório de Biodinâmica do Departamento de Educação Física, Instituto de Biociências da UNESP - Universidade Estadual Paulista, campus de Rio Clare SP, à temperatura ambiente de $25{ }^{\circ} \mathrm{C}$ e fotoperíodo de 12 horas de claro/escuro, em gaiolas de polietileno (cinco ratos por gaiola), alimentados com ração balanceada Purina ${ }^{\circledR}$ e água "ad libitum".

\section{Instrumentos e Procedimentos}

\section{Indução do diabetes}

Para indução do diabetes, os ratos, após jejum de 12 horas, receberam aloxana monoidratada Sigma (32 mg/kg de peso corporal) dissolvida em tampão citrato $0.01 \mathrm{M}, \mathrm{pH} 4.5$ injetada na veia dorsal peniana (Luciano \& Lima, 1997). Após este procedimento, os animais foram colocados em gaiolas coletivas (cinco ratos por gaiola) recebendo, nas primeiras 24 horas, uma solução de água e glicose (15\%) para evitar as complicações da hipoglicemia aloxânica (Lenzen, 2007). Sete dias após a administração da droga foi realizada uma avaliação da glicemia, a fim de se comprovar o estado diabético dos animais. Foram considerados diabéticos apenas aqueles animais que apresentaram valores de glicemia entre 200 e $600 \mathrm{mg} / \mathrm{dL}$. Os animais que apresentaram valores glicêmicos fora desse intervalo foram descartados. Como controles, foram usados ratos injetados com veículo (tampão citrato), para simulação do estresse sofrido pelos animais aloxânicos.

\section{Grupos experimentais}

Os ratos foram distribuídos aleatoriamente, de acordo com a presença do diabetes e/ou realização do treinamento físico, nos seguintes grupos: Controle Sedentário (CS) - ratos não submetidos ao treinamento físico; Controle Treinado (CT) - ratos submetidos ao proto colo de treinamento físico de natação; Diabéticos Sedentários (DS) - ratos diabéticos aloxâ nicos não submetidos ao treinamento físico e Diabéticos Treinados (DT) - ratos diabéticos aloxânicos e submetidos ao protocolo de treinamento físico de natação.

\section{Adaptação ao meio líquido}

Todos os animais foram adaptados ao meio líquido, sendo os Grupos Treinados nos 15 dias que antecederam o início do período de treinamento e os Grupos Sedentários nos 15 dias que antecederam os testes de esforço para determinação da Máxima Fase Estável de Lactato. A adaptação consistiu em manter o animal na água rasa a uma temperatura de $30 \pm 1$ ${ }^{\circ} \mathrm{C}$, por 60 minutos, cinco dias por semana, durante duas semanas. O propósito da adaptação foi reduzir o estresse dos animais frente ao exercício físico realizado na água (Voltarelli, Gobatto, \& Mello, 2002).

\section{Máxima Fase Estável de Lactato}

A Máxima Fase Estável de Lactato (MFEL) equivale à mais alta concentração de lactato sanguíneo, onde sua entrada na circulação é compensada pela remoção durante exercícios com cargas constantes (Heck et al., 1985). Sua determinação tem sido útil na prescrição de exercícios e na avaliação do condicionamento aeróbio, tanto de seres humanos como de modelos animais. Recentemente, nosso grupo 
de pesquisa descreveu um protocolo para a determinação da MFEL para ratos durante exercício de natação (Gobatto et al., 2001; Mota et al., 2008), o qual foi utilizado por nós no presente estudo.

Para tal determinação, os animais previamente adaptados ao meio líquido foram submetidos a vários testes de natação suportando sobrecargas constantes e crescentes em relação ao peso corporal ( $4 \%, 5 \%$ e $6 \%$ ), em dias separados, com intervalos de 48 horas entre eles, até que não fosse mais observada estabilização das concentrações de lactato sanguíneo durante a sessão de exercício.

Cada teste consistiu de 25 minutos de natação contínua com sobrecarga atada ao dorso do animal (mochila contendo chumbo) e de coleta sanguínea $(25 \mu \mathrm{L})$ utilizando capilares heparinizados e calibrados, por meio de pequeno e único corte na extremidade da cauda do animal, a cada 5 minutos, para a determinação das concentrações de lactato sanguíneo. A concentração de lactato sanguíneo foi determinada pelo método enzimático, proposto por Engels e Jones (1978). O critério de estabilização empregado foi a diferença entre os valores de concentração de lactato sanguíneo, entre 10 e 25 minutos de exercício, igual ou inferior a 1.0 mM (Gobatto et al., 2001).

\section{Protocolo de treinamento}

$\mathrm{O}$ protocolo de treinamento consistiu de exercício de natação por 60 minutos diários, cinco dias por semana, durante oito semanas ininterruptas. Após um período de adaptação de 15 dias, os animais nadaram suportando uma sobrecarga de chumbo atada ao tórax, equivalente à intensidade de $90 \%$ da transição metabólica individual obtida pelo teste da MFEL ao início do experimento. As sessões de natação foram realizadas em recipiente de amianto (100 cm de comprimento, $70 \mathrm{~cm}$ de largura e $60 \mathrm{~cm}$ de altura), contendo água numa profundidade de $40 \mathrm{~cm}$ com o intuito de evitar que os animais apoiassem a cauda no fundo do recipiente e/ou saltassem. A temperatura da água foi controlada por meio de um aquecedor elétrico e mantida em $31^{\circ} \mathrm{C} \pm 1{ }^{\circ} \mathrm{C}$.

\section{Avaliações gerais}

Todos os animais tiveram peso corporal e ingestões alimentar e hídrica registadas uma vez por semana e os resultados foram analisados através do cálculo das áreas sob as curvas dessas variáveis ao longo de todo o experimento pelo método trapezoidal - software ORIGIN $^{\circledR} 6.0$ (Mathews, Altman, Campbell, \& Royston, 1990).

\section{Teste de tolerância à glicose - TTGo}

O TTGo foi realizado com os animais na última semana do experimento, após um período de 15 horas de jejum. Uma primeira coleta de sangue foi feita por meio de um único corte na extremidade da cauda do animal (tempo 0). Em seguida, uma solução de glicose a $20 \%$ ( $2 \mathrm{~g} / \mathrm{kg}$ de peso) foi administrada aos ratos através de sonda gástrica de polietileno. Amostras de sangue foram coletadas após 30, 60 e 120 minutos com capilares heparinizados e calibrados $(25 \mu \mathrm{L})$, visando a determinação das concentrações de glicose. As concentrações de glicose sanguínea foram determinadas pelo método glicose-oxidase, utilizando kits comerciais $\left(\right.$ Laborlab $\left.{ }^{\circledR}\right)$. Os resultados foram analisados através da determinação das áreas sob as curvas glicêmicas durante o teste pelo método trapezoidal - software ORIGIN ${ }^{\circledR} 6.0$ (Mathews et al., 1990).

\section{Obtenção de material biológico}

Ao final do experimento e transcorridas 48 horas após a última avaliação "in vivo" e em repouso, os animais foram anestesiados com $\mathrm{CO}_{2}$ e subsequentemente exsanguinados para que fossem coletadas amostras de sangue visando a separação do soro para dosagens de: (i) glicose, pelo método enzimático colorimétrico da glicose oxidase-peroxidase (Henry, O'Connell, Smith, Chanmugam, \& Rajagopa, 1974); e (ii) insulina, por radioimuno-ensaio (Herbert, Lau, Gotlieb, \& Bleicher, 1965). O pâncreas foi extirpado por inteiro para pesagem, macerado e utilizado para a determinação 
da concentração de insulina (Malaisse, Malaisse-Lagae, \& Wright, 1967).

\section{Análise Estatística}

O tratamento estatístico foi realizado primeiramente pela análise de normalidade da distribuição dos dados (Shapiro-Wilk), seguido de análise de variância (ANOVA) de uma entrada, com aplicação de teste "post-hoc" de Bonferroni, quando apropriado. Em todos os casos, o nível de significância foi estabelecido em $5 \%$.

\section{RESULTADOS}

\section{Resultados após a indução ao diabetes}

Com o intuito de adotar uma linha de base em relação ao peso corporal e ingestões hídrica e alimentar entre os grupos, logo após a indução ao diabetes, os devidos dados foram analisados. Observou-se que os grupos diabéticos apresentaram valores de peso corporal meno- res em relação aos grupos controles. No que refere às ingestas alimentar e hídrica, os grupos diabéticos apresentaram valores superiores aos grupos controles, mas os grupos Diabéticos (DS e DT) assim como os Controles (CS e CT) não diferiram entre si (Tabela 1).

No que se refere à determinação da MFEL dos animais ao início do experimento, não foram observadas diferenças entre os grupos (Tabela 2).

Tabela 2 .

Concentração sanguínea de lactato $(\mathrm{Mmol} / \mathrm{L})$ e sobrecarga (\% do peso corporal) equivalente à Máxima Fase Estável de Lactato no início do experimento

\begin{tabular}{lcc}
\hline & $\begin{array}{c}\text { Controle } \\
\mathrm{M} \pm \mathrm{DP}\end{array}$ & $\begin{array}{c}\text { Diabético } \\
\mathrm{M} \pm \mathrm{DP}\end{array}$ \\
\hline Lactacidemia & $4.0 \pm 0.6$ & $4.5 \pm 0.5$ \\
Sobrecarga & $6.4 \pm 0.4$ & $5.5 \pm 0.4$ \\
\hline
\end{tabular}

Nota: $n=10$ animais por grupo; Não se verificaram diferenças significativas entre grupos $(p>.05)$

Tabela 1.

Peso corporal (gramas), ingestão hídrica ( $\mathrm{ml} / 100 \mathrm{~g}$ de rato) e alimentar ( $\mathrm{g} / 100 \mathrm{~g}$ de rato) após a indução ao diabetes

\begin{tabular}{lcccc}
\hline & CS $(n=10)$ & $\mathrm{CT}(n=10)$ & $\mathrm{DS}(n=10)$ & $\mathrm{DT}(n=10)$ \\
& $\mathrm{M} \pm \mathrm{DP}$ & $\mathrm{M} \pm \mathrm{DP}$ & $\mathrm{M} \pm \mathrm{DP}$ & $\mathrm{M} \pm \mathrm{DP}$ \\
\hline Peso corporal & $439.96 \pm 31.18$ & $468.85 \pm 42.99$ & $340.14 \pm 39.57^{\mathrm{a}}$ & $342.20 \pm 34.23^{\mathrm{a}}$ \\
Ingestão hídrica & $9.51 \pm 2.01$ & $6.33 \pm 0.47$ & $61.20 \pm 23.54^{\mathrm{a}}$ & $57.33 \pm 9.69^{\mathrm{a}}$ \\
Ingestão alimentar & $6.33 \pm 0.26$ & $5.50 \pm 0.39$ & $12.06 \pm 4.21^{\mathrm{a}}$ & $15.07 \pm 2.57^{\mathrm{a}}$ \\
\hline
\end{tabular}

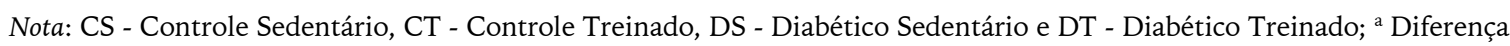
significativa $(p<.05)$ em relação aos grupos controles

\section{Resultados ao final de oito semanas de experimento}

Os animais pertencentes aos grupos diabéticos apresentaram redução do peso corporal durante o experimento quando comparados aos controles. O grupo DT mostrou redução menos acentuada se comparado ao DS (Figura 1). Em relação às ingestões hídrica e alimentar, os grupos diabéticos apresentaram valores maiores quando comparados aos grupos controles (Figuras 2 e 3, respetivamente).

Em relação ao teste de tolerância à glicose oral (TTGo), a área sob a curva de glicose sérica dos animais diabéticos mostrou-se supe-

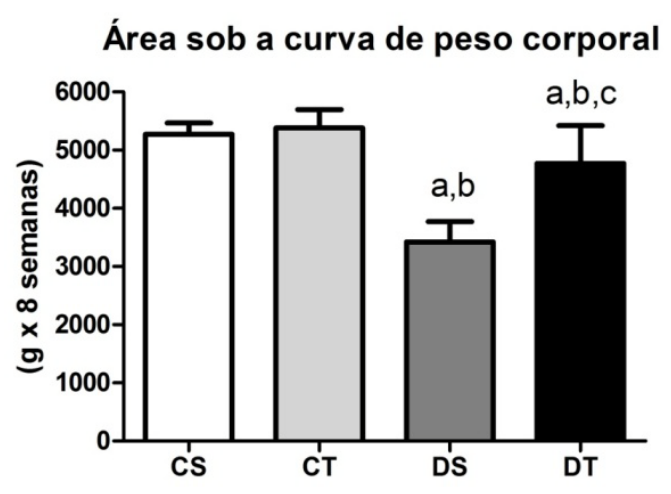

Figura 1. Área sob a curva de peso corporal durante oito semanas de experimento $(\mathrm{M} \pm \mathrm{DP})$; Diferença estatística (ANOVA, $p<.05$ ): a - diferente de CS, $\mathrm{b}$ - diferente de CT, $\mathrm{c}$ - diferente de DS 
28 | L. Pereira de Moura, R.J. Gomes, J.A. Leme, F.A. Voltarelli, C. Ribeiro, R. Ferreira de Moura, et al.

rior se comparados aos controles. Quando a comparação foi feita apenas entre os grupos diabéticos, os animais sedentários apresentaram maiores valores de área sob a curva de glicose em relação aos animais treinados. (Figura 4).

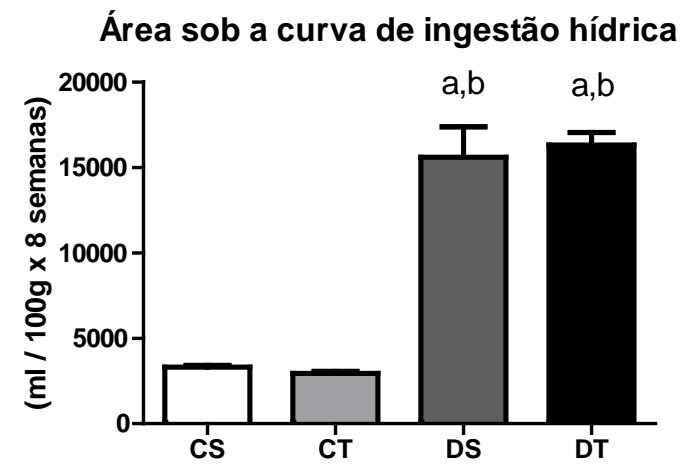

Figura 2. Área sob a curva de ingestão hídrica durante as oito semanas de experimento $(\mathrm{M} \pm \mathrm{DP})$;

Diferença estatística (ANOVA, $p<.05$ ):

$\mathrm{a}$ - diferente de CS, $\mathrm{b}$ - diferente de CT

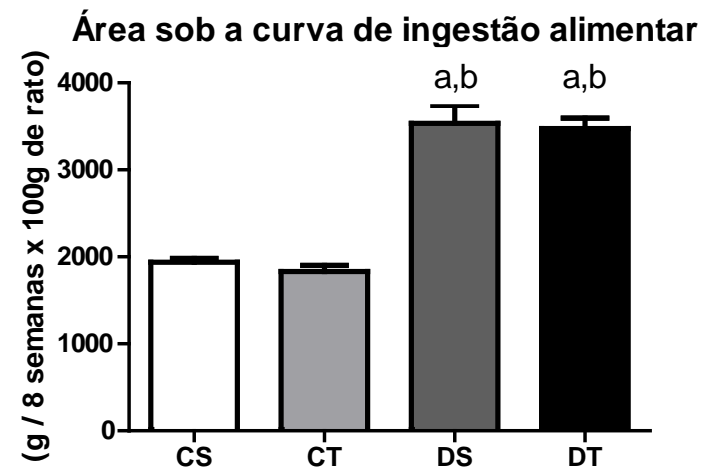

Figura 3. Área sob a curva de ingestão alimentar durante as oito semanas de experimento $(\mathrm{M} \pm \mathrm{DP})$;

Diferença estatística (ANOVA, $p<.05$ ): a - diferente de CS, $\mathrm{b}$ - diferente de CT
Área sob a curva glicêmica no TTGo

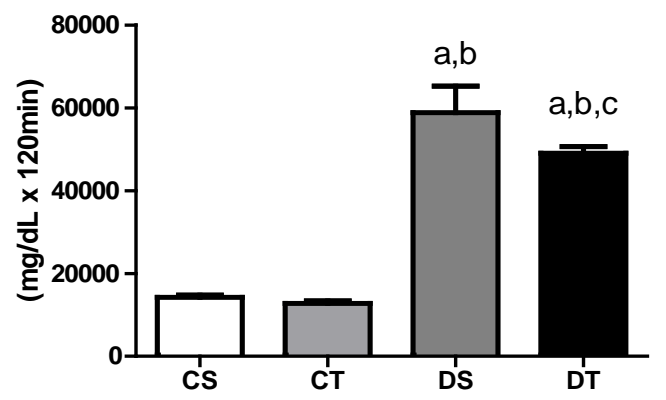

Figura 4. Área sob a curva glicêmica durante TTGo ao final das oito semanas de experimento $(M \pm D P)$;

Diferença estatística (ANOVA, $p<.05)$ : a - diferente de CS, $\mathrm{b}$ - diferente de CT, $\mathrm{c}$ - diferente de DS

\section{Insulina Pancreática}

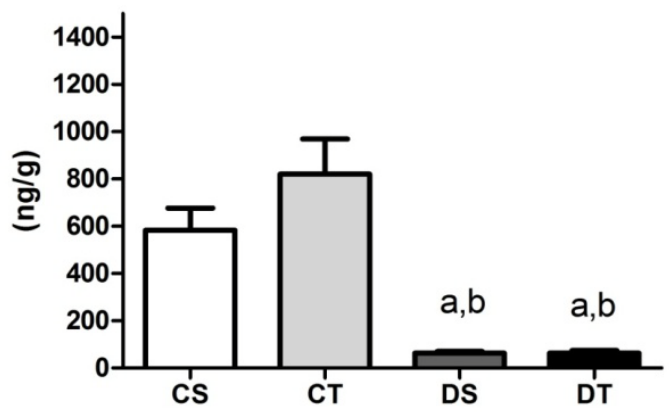

Figura 5. Insulina Pancreática ao final das oito semanas de experimento $(\mathrm{M} \pm \mathrm{DP})$; Diferença estatística (ANOVA, $p<.05$ ): $\mathrm{a}$ - diferente de CS, $\mathrm{b}$ - diferente de CT

A insulina sérica dos animais diabéticos, ao final do experimento, mostrou-se mais baixa em relação aos controles (Tabela 3). A glicemia dos ratos diabéticos foi superior à dos controles, sendo que o grupo DT apresentou redução em comparação ao DS (Tabela 3).

Tabela 3.

Insulina $(\mu U / L)$ e glicemia séricas $(\mathrm{mg} / \mathrm{dL})$ ao final do experimento.

\begin{tabular}{lcccc}
\hline & $\mathrm{CS}(n=10)$ & $\mathrm{CT}(n=10)$ & $\mathrm{DS}(n=10)$ & $\mathrm{DT}(n=10)$ \\
& $\mathrm{M} \pm \mathrm{DP}$ & $\mathrm{M} \pm \mathrm{DP}$ & $\mathrm{M} \pm \mathrm{DP}$ & $\mathrm{M} \pm \mathrm{DP}$ \\
\hline Insulina & $663.70 \pm 223.10$ & $821.20 \pm 332.60$ & $63.40 \pm 16.30^{\mathrm{a}}$ & $66.20 \pm 26.10^{\mathrm{a}}$ \\
Glicose & $102.00 \pm 10.00$ & $105.00 \pm 9.00$ & $323.00 \pm 12.00^{\mathrm{a}}$ & $301.00 \pm 13.00^{\mathrm{a}, \mathrm{b}}$ \\
\hline
\end{tabular}

Nota: CS - Controle Sedentário, CT - Controle Treinado, DS - Diabético Sedentário e DT - Diabético Treinado; Diferença significativa $(p<.05)$ em relação: ${ }^{\text {a }}$ aos grupos controles, ${ }^{\mathrm{b}}$ ao grupo DS 
Os animais pertencentes aos grupos diabéticos apresentaram concentrações de insulina pancreáticas mais baixas se comparados aos grupos controles (Figura 5).

\section{DISCUSSÃO}

De acordo com Lenzen (2007), a administração de aloxana em ratos provoca quadro diabético semelhante ao encontrado em humanos, incluindo hiperglicemia, perda de peso, polifagia e polidpisia. No presente estudo, a administração de aloxana através da veia peniana dorsal do animal seguiu o procedimento preconizado por Luciano e Lima (1997), o qual se mostrou bastante eficiente na indução do quadro diabético.

No presente estudo, verificou-se que os animais pertencentes aos grupos diabéticos apresentaram perda acentuada de peso em relação aos controles, após a indução do diabetes como no final do experimento. Tal fato pode ser devido, pelo menos em parte, ao hipoinsulinismo, o qual conduz a um estado catabólico de proteínas e gorduras, além de implicar uma glicosúria associada à poliúria (Kimbal, Vary, \& Jefferson, 1994).

A American Diabetes Association (2006) enfatiza que a falta de controle da homeostase glicêmica pode levar, em longo prazo, a diversas disfunções, tais como: retinopatia, nefropatia, neuropatias periféricas autonômicas, cetoacidose e síndrome hiperosmolar não-cetótica. Esta última gera aumento excessivo da ingestão hídrica, fato observado em nosso estudo desde o início até o final do experimento e que não foi revertido pelo treinamento físico. No presente estudo, foram utilizados animais apresentando características do diabetes tipo 1 e não tratados com insulina. A hipoinsulinemia eleva de forma excessiva a ingestão alimentar, pois credita-se à insulina uma inibição da atividade neuronal no núcleo arqueado do hipotálamo, o qual coexpressa tanto neuropeptídeo Y como peptídeos relacionados à cepa agouti (AGRP), moléculas estas que potencialmente estimulam o consumo alimentar (Asakawa, Toyoshima, Inoue, \& Koizume, 2007). Outra hipótese para a elevação excessiva da ingestão alimentar, observada no presente estudo, pode ser a ocorrência de hipoleptinemia, tanto em termos de concomitância com a hipoinsulinemia, quanto de sobreposição (Hidaka et al., 2001).

Um estudo realizado por Dong et al. (2006) mostrou que o hormônio leptina aumentou a expressão de neuropeptídeo Y (NPY) bem como reduziu a expressão do hormônio estimulador dos melanócitos ( $\alpha-\mathrm{MSH})$ em ratos diabéticos induzidos por estreptozitocina. Além disto, em ratos knock-out para grelina, a indução de diabetes não aumentou a expressão de NPY. Ainda, a utilização deficiente de glicose pelas células do núcleo ventromedial do hipotálamo também contribui para a hiperfagia diabética. Quando a utilização de glicose no núcleo ventromedial do hipotálamo é baixa, sua atividade é diminuída, acarretando em sensação de fome. Os animais pertencentes aos grupos diabéticos, em nosso estudo, apresentaram hiperfagia diabética desde o início do experimento até seu final, ao passo que o treinamento físico realizado não foi capaz de reverter essa condição.

A determinação da MFEL foi necessária, no início do experimento, para que a intensidade do esforço durante os treinos fosse estabelecida. A MFEL equivale à mais alta concentração sanguínea de lactato onde sua entrada na circulação é equivalente à remoção durante o exercício com carga constante (Heck et al., 1985). Há algum tempo, nosso grupo de pesquisa (Gobatto et al., 2001) desenvolveu um estudo para a determinação da MFEL de ratos sadios durante o exercício de natação e permanece utilizando-o extensivamente. Nesse estudo, a MFEL foi obtida na carga de $6 \%$ do peso corporal à concentração sanguínea de lactato de $5.5 \mathrm{mmol} / \mathrm{l}$ de sangue. O mesmo foi observado no presente estudo. Ainda, Oliveira, Luciano, Gomes-Marcondes e Mello (2007), determinaram a transição aeróbia/anaeróbia de ratos diabéticos pelo método do lactato mínimo e encontraram valores médios de lactato em $6.9 \mathrm{mmol} / \mathrm{L}$, os quais não corroboram 
os resultados obtidos no presente estudo, onde o limiar foi encontrado em valores próximos de $4.5 \mathrm{mmol} / \mathrm{L}$. Isso pode ser explicado, pelo menos em parte, pela utilização de diferentes protocolos de teste (MFEL versus lactato mínimo). A MFEL utiliza vários testes, realizados em dias diferentes, com intensidades distintas, mas fixas a cada teste $(4 \%, 5 \%$ e $6 \%)$, até que se atinja a estabilização da concentração de lactato sanguíneo entre o $10^{\circ}$ e o $25^{\circ}$ minuto de exercício (Gobatto et al., 2001).

Para diagnosticar o diabetes, em seres humanos, é realizado o teste de tolerância à glicose oral (TTGo), o qual fornece indicativos quanto à tolerância à glicose. Pacientes diabéticos apresentam menor tolerância à glicose, ao passo que a prática de atividade física regular pode amenizar esse quadro. Em relação aos resultados obtidos no presente estudo, observou-se intolerância à glicose nos grupos diabéticos se comparados aos controles. Por outro lado, os animais pertencentes ao grupo DT apresentaram maior tolerância à glicose em relação aos animais diabéticos sedentários, mostrando o efeito positivo do exercício físico sobre a doença (Eriksson, Taimela, \& Koivisto, 1997; Luciano \& Mello, 1998). Em contrapartida, em estudo desenvolvido por Oliveira et al. (2007), onde ratos diabéticos aloxânicos foram treinados por natação na intensidade da transição metabólica determinada pelo teste do lactato mínimo, nenhum efeito do treinamento sobre a tolerância à glicose foi observado. Isso reforça a importância do estabelecimento da adequada intensidade do esforço durante o exercício no treinamento de diabéticos.

Numa tentativa de ampliar as informações sobre os efeitos do treinamento individualizado em intensidade equivalente a $90 \%$ da MFEL, analisamos a concentração de insulina no pâncreas dos animais ao final do experimento. Os resultados referentes à concentração de insulina pancreática dos animais diabéticos (DS e DT) confirmam que a administração de aloxana resultou em destruição das células beta, conduzindo à insulinopenia (Lenzen, 2007). O treinamento físico aplicado para os animais diabéticos não foi capaz de alterar esse quadro.

Resumidamente, o treinamento físico em intensidade equivalente a $90 \%$ da MFEL, embora não tenha alterado os estoques pancreáticos de insulina, foi eficaz em melhorar a homeostase glicêmica, fazendo com que os tecidos utilizem a glicose como substrato energético na ausência de insulina e auxiliar na manutenção do peso corporal dos animais diabéticos, evitando a perda de peso acentuada.

\section{CONCLUSÕES}

Tomados em conjunto, os resultados do presente estudo mostram que o treinamento físico realizado a $90 \%$ da intensidade da Máxima Fase Estável de Lactato:

(i) Não foi capaz de alterar os estoques pancreáticos de insulina, tanto de ratos diabéticos, como eutróficos;

(ii) Atenuou a perda de peso corporal dos animais diabéticos;

(iii) Foi eficaz em melhorar a homeostase glicêmica dos ratos diabéticos.

\section{Agradecimentos:}

Os autores gostariam de agradecer o indispensável apoio dos técnicos do Laboratório de Biodinâmica do Departamento de Educação Física da UNESP Campus Rio Claro: Clarice Sibuya e José Roberto Rodrigues.

\section{Conflito de Interesses:}

Nada a declarar.

\section{Financiamento:}

Os autores declaram que este teve o apoio financeiro do Conselho Nacional de Desenvolvimento Científico e Tecnológico - CNPq, da FUNDUNESP e da Fundação de Amparo à Pesquisa do Estado de São Paulo - FAPESP.

\section{REFERÊNCIAS}

American Diabetes Association (2006). Diagnosis and classification of diabetes mellitus. Diabetes Care, 29, S43-S48. doi: 10.2337/diacare.27.20 07.S5

Asakawa, A., Toyoshima, M., Inoue, K., \& Koizume, A. (2007). Ins2Akita mice exhibit hyperphagia 
and anxiety behavior via the melanocortin system. International Journal of Molecular Medicine, 19, 649-652.

Beneke, R. (2003). Methodological aspects of maximal lactate steady state-implications for performance testing. European Journal of Applied Physiology, 89, 95-99. doi: 10.1007/s00421-0020783-1

Billat, V. L. Siverent, P., Py, G., Korallsztein, J.-P., \& Mercier, J. (2003). The concept of maximal lactate steady state: A bridge between biochemistry, physiology and sport science. International Journal of Sports Medicine, 33, 407-426.

Boquist, L. (1977). The endocrine pancreas in early alloxan diabetes. Including study of the alloxan inhibitory effect of feeding and some hexoses. Acta Pathologica et Microbiologica Scandinavica, 85A, 219-229.

Chan, J. C., Malik, V., \& Jia, W. (2009). Diabetes in Asia: Epidemiology, risk factors, and pathophysiology. Journal of the American Medical Association, 301, 2129-2140. doi: 10.1001/jama.20 09.726

Cortright, R. N., \& Dohm, G. L. (1997). Mechanisms by which insulin and contraction stimulate glucose transport. Canadian Journal Applied Physiology, 22, 519-530.

Dong, J., Peeters, T. L., De Smet, B., Moechars, D., Delporte, C., Vanden Berghe, P., ... Depoortere, I. (2006). Role of endogenous ghrelin in the hyperphagia of mice with strep-tozotocininduced diabetes. Endocrinology, 147, 2634-2642.

Engels, R. C., \& Jones, J. B. (1978). Causes and elimination of erratic blanc in enzymatic metabolic assays involving the use of NAD in alkaline hydrazine buffers: Improved conditions for assay of L-glutamate, L-lactate and other metabolites. Analytical Biochemistry, 88, 475-484.

Eriksson, J., Taimela, S., \& Koivisto, V. A. (1997). Exercise and the metabolic syndrome. Diabetologia, 40, 125-135.

Gobatto, C. A., de Mello, M. A., Sibuya, C. Y., de Azevedo, J. R., dos Santos, L. A., \& Kokubun, E. (2001). Maximal lactate steady state in rats submitted to swimming exercise. Comparative Biochemistry and Physiology A, Molecular \& Integrative Physiology, 130, 21-27. doi: 10.1016/S10956433(01)00362-2

Harnish, C. R., Swensen, T. C., \& Pate, R. P. (2001). Methods for estimating the maximal lactate steady state in trained cyclists. Medicine $\mathcal{E}$ Science in Sports \& Exercise, 33, 1052-1055.
Heck, H., Mader, A., Hess, G., Mücke, S., Müller, R., \& Hollmann, W. (1985). Justification of the 4 $\mathrm{mM} / 1$ lactate threshold. International Journal of Sports Medicine, 6, 117-130.

Henry, R. J., O'Connell, R. F., Smith, E. R., Chanmugam, G., \& Rajagopa, A. K. (1974). Energy spectrum of $\mathrm{H}$ - in a strong magnetic field. The American Physical Society, 9, 329-331.

Herbert, V., Lau, K. S., Gotlieb, C. W., \& Bleicher, S. T. (1965). Coated charcoals immunoassay of insulin. Journal of Clinical Endocrinology and Metabolism, 25, 1375-1384.

Hidaka, S., Yoshimatsu, H., Kondou, S., Oka, K., Tsuruta, Y., Sakino, H., ... Sakata, T. (2001). Hypoleptinemia, but not hypoinsulinemia, induces hyperphagia in streptozotocin-induced diabetic rats. Journal of Neurochemistry, 77, 9931000 .

Kimball, S. R., Vary, T. C., \& Jefferson, L. S. (1994). Regulation of protein synthesis by insulin. Annual Review of Physiology, 56, 321-348.

Lenzen, S. (2007). Alloxan and streptozotocin diabetes. In E. Peschke (Ed.), Endokrinologie III Vorträge im Rahmen des Projektes 'Zeitstrukturen endokriner Systeme'. [Endocrinology III lectures within the 'time structures of endocrine systems' project framework] (pp. 119-138). Stuttgart: Verlag.

Luciano, E., \& Lima, F. B. (1997). Metabolismo de ratos diabéticos treinados submetidos ao jejum e ao exercício. Revista de Ciências Biomédicas, 18, 47-60.

Luciano, E., \& Mello, M. A. (1998). Atividade física e metabolismo de proteínas em músculo de ratos diabéticos experimentais. Revista Paulista de Educação Física, 2, 202-209.

Luciano, E., Carneiro, E. M., Carvalho, C. R., Carvalheira, J. B. C., Peres, S. B., Reis, M. A., ... Velloso, L. A. (2002). Endurance training improves responsiveness to insulin and modulates insulin signal transduction through the phosphatidylinositol 3-kinase/Akt-1 pathway. European Journal of Endocrinology, 147, 149-157. doi: 10.1530/eje.0.1470149

Mader, A., \& Heck, H. (1986). A theory of metabolic origin of the anaerobic threshold. International Journal of Sports Medicine, 7, 45-65.

Malaisse, W. J., Malaisse-Lagae, F., \& Wright, P. H. (1967). Effect of fasting upon insulin secretion in the rat. American Journal of Physiology, 213, 843-848.

Mathews, J. N. S., Altman, D. G., Campbell, M. J., \& Royston, P. (1990). Analysis of serial meas- 
32 | L. Pereira de Moura, R.J. Gomes, J.A. Leme, F.A. Voltarelli, C. Ribeiro, R. Ferreira de Moura, et al.

urements in medical research. British Medical Journal, 27, 230-235.

Mota, C. A., Ribeiro, C., Araujo, G. G., Araújo, M. B., Manchado, F. B., Voltarelli, F. A., ... Mello, M. A. (2008). Exercise training in the aerobic/ anaerobic metabolic transition prevents glucose intolerance in alloxan-treated rats. BMC Endocrine Disorders, 8, 11-24. doi: 10.1186/1472-682 3-8-11

Oliveira, C. A., Luciano, E., Gomes-Marcondes, M. C., \& Mello, M. A. (2007). Effects of swimming training at the intensity equivalent to aerobic/ anaerobic metabolic transition in alloxan diabetic rats. Journal of Diabetes Complications, 21 (4), 258-264. doi: 10.1016/j.jdiacomp.2006. 07.007

Sabetsky, V., \& Ekblom, J. (2010). Insulin: A new era for an old hormone. Pharmacological Research, 61, 1-4. doi: 10.1016/j.phrs.2009.07.010
Szkudelski, T. (2001). The mechanism of alloxan and streptozotocin action in b cells of the rat pancreas. Physiological Research, 50, 536-546.

The Expert Committee on the Diagnosis and Classification of Diabetes Mellitus (1997). Report of the Expert Committee on the diagnosis and classification of diabetes mellitus. Diabetes Care, 20, 1183-1197.

Voltarelli, F. A., Gobatto, C. A., \& Mello, M. A. (2002). Determination of anaerobic threshold in rats using the lactate minimum test. Brazilian Journal of Medical and Biological Research, 35, 1389-1394.

White, M. F. (1997). The insulin signaling system and the IRS proteins. Diabetologia, 40, 2-17.

Wrenshall, G. A., Bogoch, A., \& Ritchie, R. (1952). Extractable insulin of pancreas: Correlation with pathological and clinical findings in diabetic and nondiabetic cases. Diabetes, 1, 87-107.

(cc) EY-No Todo o conteúdo da revista Motricidade está licenciado sob a Creative Commons, exceto quando especificado em contrário e nos conteúdos retirados de outras fontes bibliográficas. 